\title{
Collapse Transition of Two-Dimensional Flexible and Semiflexible Polymers *
}

\author{
Haijun Zhou ${ }^{1}$, Jie Zhou ${ }^{1}$, Zhong-Can Ou-Yang ${ }^{1}$, and Sanjay Kumar ${ }^{2}$ \\ ${ }^{1}$ Institute of Theoretical Physics, the Chinese Academy of Sciences, Beijing 100080, China and \\ ${ }^{2}$ Department of Physics, Banaras Hindu University, Varanasi 221 005, India
}

(Dated: September 6, 2018)

\begin{abstract}
The nature of the globule-coil transition of surface-confined polymers has been an issue of debate. Here this 2D collapse transition is studied through a partially directed lattice model. In the general case of polymers with positive bending stiffness $(\Delta>0)$, the collapse transition is first-order; it becomes second-order only in the limiting case of zero bending stiffness $(\Delta \equiv 0)$. These analytical results are confirmed by Monte Carlo simulations. We also suggest some possible future experiments.
\end{abstract}

PACS numbers: 82.35.Lr, 05.50.+q, 64.60.Cn, 82.35.Gh

Keywords: collapse, globule-coil transition, semiflexible, lattice polymer

The collapse (globule-coil) transition of a selfattracting chain is one of the fundamental problems in polymer physics [1, 2, 3]. Being deeply connected with biophysical problems such as DNA condensation, chromatin organization, and protein folding, the collapse transition has also biological relevance. Generally speaking, this transition is caused by the competition between monomer-monomer attraction and configurational entropy: Formation of contacts lowers the energy, but it requires monomers to be aligned and close to each other, thereby decreasing the polymer's degrees of configurational freedom. When the monomer-monomer attraction is much stronger than thermal energy, the polymer takes globular conformations to maximize the number of contacts. At the other limit of high temperatures, the polymer is in completely disordered coil states with maximal entropy. At zero external force, the collapse transition occurs at a so-called $\theta$-temperature [1].

The exact nature of the collapse transition, however, is not yet completely settled, despite decades of extensive efforts [1, 2, 3]. This transition can be studied by mapping to the tricritical point of the $\phi^{4}-\phi^{6} O(n)$ field theory at the $n \rightarrow 0$ limit $[4,[5,[6]$, and is expected to be second-order in 2D and beyond (see, e.g., 7]); furthermore, exponents of the temperature-driven collapse and adsorption of a 2D polymer grafted on a linear boundary was obtained by analytical calculations [8, 9]. However, experimentally observed collapse transitions of both relatively flexible [10] and semiflexible [11] 3D polymers are more like first-order transitions. Recent Monte Carlo (MC) simulation [12] is also in favor of the latter interpretation.

With the advancement in single-molecule force manipulation methods, now the globule-coil transition can also be induced by external stretching [13, 14]. According to mean-field theory [15] the force-induced collapse transition is first-order in all dimensions. This claim is con-

${ }^{*}$ Citation information: H. Zhou, J. Zhou, Z.-C. Ou-Yang, and S. Kumar, Phys. Rev. Lett. 97, 158302 (2006). firmed by MC simulation in 3D [16], while in 2D the transition is argued to be second-order by MC simulation [16] and scaling analysis [17, 18].

In order to understand the collapse transition more deeply, here we investigate a $2 \mathrm{D}$ partially directed lattice model of a polymer chain which is exactly solvable (Fig. 1). As the free energy density of this model system can be calculated precisely, we are able to draw definite conclusions concerning the nature of the collapse transition. We find that the collapse transition is second-order for a polymer with exactly zero bending stiffness, while it changes to be first-order when the bending stiffness $\Delta$ is non-zero. Therefore bending energies have a dramatic effect on the cooperativity of the 2D globule-coil transition. We also substantiate our predictions by performing extensive MC simulations. It becomes experimentally feasible to confine polymers to $2 \mathrm{D}$ mobile surfaces (see, e.g., [19]), therefore, future experiments will be able to verify the theoretical predictions. The present work is also directly applicable to the biology-related problem of protein $\beta$-sheet unfolding.

The model.- The 2D partially directed polymer of $N$ identical units on a square lattice is shown in Fig. 1. The length of the bond connecting two consecutive monomers $i$ and $i+1$ is fixed to $a_{0}$, while the direction of bond in $-\mathbf{z}_{0}$ is prohibited. If any two monomers $i$ and $i+m(m \geq 3)$ occupy nearest neighboring lattice sites, an attractive en-

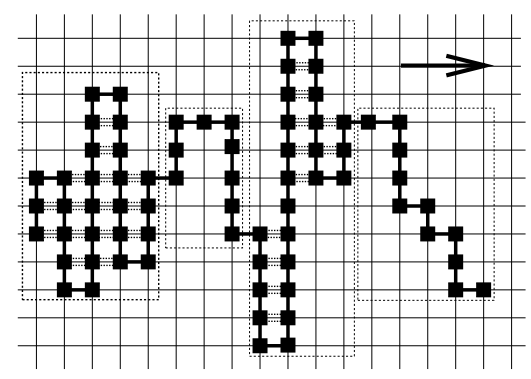

FIG. 1: The 2D lattice polymer model with contacting interaction, bending stiffness, and external stretching. The arrow shows the $\mathbf{z}_{0}$ direction. 
ergy of magnitude $\epsilon$ is gained. Usually real polymers are semiflexible, we associate an energy penalty of magnitude $\Delta$ to each local direction change of the chain $[20,21]$. For this model, only the special case of zero bending stiffness has been studied analytically [18, 22, 23]. The results of the present paper suggest that, in the general case of $\Delta>0$ the properties of the polymer will be dramatically different from these earlier predictions [18, 22, 23].

Partition functions. - To calculate the free energy density of the polymer, a given configuration of the $2 \mathrm{D}$ chain is divided into a linear sequence of $\beta$-sheet segments and coil segments [24]. A $\beta$-sheet segment is defined as a folded segment of $n_{\beta} \geq 2$ consecutive columns, in which contacting interactions exist between any two adjacent ones. Two consecutive $\beta$-sheet segments are separated by a coil segment, which is a segment of $n_{\mathrm{c}} \geq 0$ consecutive columns in which all monomers are free of contacts. For example, the configuration shown in Fig. 1 has two $\beta$-sheets and two coils. After having made such a distinction between $\beta$-sheets and coils, we proceed by first calculating the partition functions of $\beta$-sheets and coils separately.

Under the action of an external force $f$, the energy of a $\beta$-sheet of $n_{\beta}$ columns is

$$
E_{\beta}=-\epsilon \sum_{j=1}^{n_{\beta}-1} \mathrm{v}\left(l_{j}, l_{j+1}\right)-n_{\beta} f a_{0}+2\left(n_{\beta}-1\right) \Delta,
$$

where $l_{j}$ is the number of monomers in the $j$-th column of the $\beta$-sheet, and $\mathrm{v}\left(l_{j}, l_{j+1}\right)=\min \left(l_{j}, l_{j+1}\right)-1$. The partition function of a $\beta$-sheet with $n \geq 4$ monomers is

$$
Z_{\beta}(n)=\sum_{n_{\beta}=2}^{[n / 2]} \sum_{l_{j} \geq 2} \delta_{l_{1}+\cdots+l_{n_{\beta}}}^{n} p^{m} s^{2(m-1)} \prod_{j=1}^{m-1} a^{\mathrm{v}\left(l_{j}, l_{j+1}\right)},
$$

where $p=e^{f a_{0} / T}, s=e^{-\Delta / T}, a=e^{\epsilon / T}$, and $T$ is the temperature. It is easier to calculate the generating function $G_{\beta}(\zeta)$ of the partition function $Z_{\beta}(n)$ than to calculate $Z_{\beta}(n)$ directly. After some simple matrix operations [25] we find that

$$
\begin{aligned}
G_{\beta}(\zeta) & \equiv \sum_{n=4}^{\infty}(\zeta / a)^{n} Z_{\beta}(n) \\
& =\frac{p^{2} s^{2}}{a} \sum_{i, j, k=1} \frac{x_{i} x_{j} A_{i k}(\zeta) A_{j k}(\zeta) \lambda_{k}(\zeta)}{1-\left(p s^{2} / a\right) \lambda_{k}(\zeta)}
\end{aligned}
$$

In Eq. (44), $x_{j}=(\zeta / a)^{(j+1) / 2} ; \lambda_{1}(\zeta) \geq \lambda_{2}(\zeta) \geq \ldots$ are the eigenvalues of a $L \times L$ real-symmetric matrix $\Lambda(\zeta)$ with elements $\Lambda_{i j}(\zeta)=\zeta^{1+(i+j) / 2} / a^{|i-j| / 2}(i, j=1,2, \ldots, L)$; and the orthogonal matrix $A(\zeta)$ contains the eigenvectors of matrix $\Lambda(\zeta)$. The parameter $L$ should be infinity. When $\zeta>1, \lambda_{1}(\zeta)=+\infty$ and consequently $G_{\beta}(\zeta)$ is not properly defined. When $\zeta \leq 1$, all the eigenvalues of matrix $\Lambda(\zeta)$ are finite, and the value of $G_{\beta}(\zeta)$ can be calculated by Eq. (4). The point $\zeta=1$ is therefore a singular point of $G_{\beta}(\zeta)$, where it attains a finite value as long as $\lambda_{1}(1)<a /\left(p s^{2}\right)$. At this point, the first derivative of $G_{\beta}(\zeta)$ with respect to $\zeta$ is 25$]$

$$
G_{\beta}^{\prime}(1) \equiv \mathrm{d} G_{\beta}(\zeta) /\left.\mathrm{d} \zeta\right|_{\zeta=1}=\lim _{L \rightarrow \infty} \sum_{n=1}^{L} n y(n)+O(1),
$$

where $y(n)=\left(\sum_{j, k=1}^{L} A_{n j} A_{k j} x_{k} \lambda_{j} /\left(1-p s^{2} \lambda_{j} / a\right)\right)^{2}$.

The configurational energy of a coil segment is

$$
E_{c}=-n_{\mathrm{c}} f a_{0}+m_{\mathrm{c}} \Delta,
$$

where $n_{\mathrm{c}}$ and $m_{\mathrm{c}}$ are, respectively, the total number of columns and the total number of bends in the configuration. In a coil segment the value of $m_{\mathrm{c}}$ strongly depdends on the configurations. To calculate the partition function $Z_{\mathrm{c}}(n)$ of a coil segment of $n$ monomers, one needs to distinguish among four different boundary conditions [25]. The generating function $G_{\mathrm{c}}(\zeta)$ of $Z_{\mathrm{c}}(n)$ in this case is expressed as

$$
\begin{aligned}
& G_{\mathrm{c}}(\zeta) \equiv \sum_{n=0}^{\infty}(\zeta / a)^{n} Z_{\mathrm{c}}(n) \\
& =\frac{a s^{2}(a-\zeta)(a+\zeta p)}{a^{3}-\zeta a^{2}(1+p)+\zeta^{2} a p\left(1-s^{2}\right)-\zeta^{3} p^{2} s^{2}} .
\end{aligned}
$$

The divergence radius of $G_{\mathrm{c}}(\zeta)$ is easy to obtain from Eq. (8). $G_{\mathrm{c}}(\zeta)$ approaches $+\infty$ as $\zeta$ approaches this divergence radius.

The collapse transition.-Since every configuration of the polymer is of the form ...- $\beta$-c- $\beta$-c..., the generating function $G(\zeta)$ of the whole polymer's partition function $Z(N)$ can readily be written down [24, 25]:

$$
G(\zeta) \equiv \sum_{N=0}^{\infty}(\zeta / a)^{N} Z(N)=\frac{\left[1+G_{\beta}(\zeta)\right] G_{\mathrm{c}}(\zeta)}{1-G_{\beta}(\zeta) G_{\mathrm{c}}(\zeta)} .
$$

$Z(N)$ is related to the free energy density $g(f, T)$ of the polymer by $Z(N)=\exp (-N \beta g(f, T))$. Therefore, in the thermodynamic limit $N \rightarrow \infty$, the free energy density is

$$
g(f, T)=-\epsilon+T \ln \zeta_{0},
$$

where $\zeta_{0}$ is the smallest positive root in the range $0<$ $\zeta_{0}<1$ of the following equation

$$
G_{\beta}\left(\zeta_{0}\right) G_{\mathrm{c}}\left(\zeta_{0}\right)=1 .
$$

If Eq. (11) has no root in the range of $0<\zeta_{0}<1$, then $\zeta_{0}=1$. The polymer's relative extension $\ell$ along $\mathbf{z}_{0}$ is

$$
\ell \equiv-\frac{\partial \ln \zeta_{0}}{\partial \ln p}=-\frac{1}{\beta a_{0} \zeta_{0}} \frac{\partial \zeta_{0}}{\partial f}
$$

A similar expression can also be obtained for the density of contacts. For the simplicity we take the relative extension $\ell$ as our order parameter. 
When both temperature $T$ and external force $f$ are sufficiently low, Eq. (11) has no root in $0<\zeta_{0}<1$. Then $g(f, T)=-\epsilon$. The polymer is in the $\beta$-sheet phase, and $\ell \equiv 0$. There is no significant entropic contribution to the free energy density. As the temperature or the external force is elevated to certain point such that Eq. (11) is satisfied exactly at $\zeta_{0}=1$, a phase transition occurs. At this point, the $\beta$-sheet phase change to the extended coil phase. According to the work of Lifson 24], this globulecoil transition is first-order if the value of $G_{\beta}^{\prime}(1)$ in Eq. (5) is finite at the phase transition point; otherwise, it is a continuous phase transition. At the collapse phase transition point, high-precision numerical calculations reveal that $y(n)$ of Eq. (5) decays exponencially with $n$ as long as $\Delta>0$ 25. In the vicinity of $\Delta=0$ we have

$$
y(n) \sim e^{-0.88 n \Delta / \epsilon} \quad(\Delta \ll \epsilon) .
$$

Therefore, at the phase transition point $G_{\beta}^{\prime}(1)$ is finite as long as $\Delta>0$. In other words, the collapse phase transition is first-order for polymers with bending stiffness $\Delta>0$; it is second-order for polymers with exactly zero bending stiffness. It is remarkable that the cooperativity of the collapse transition can be changed by adding just a small bending stiffness.
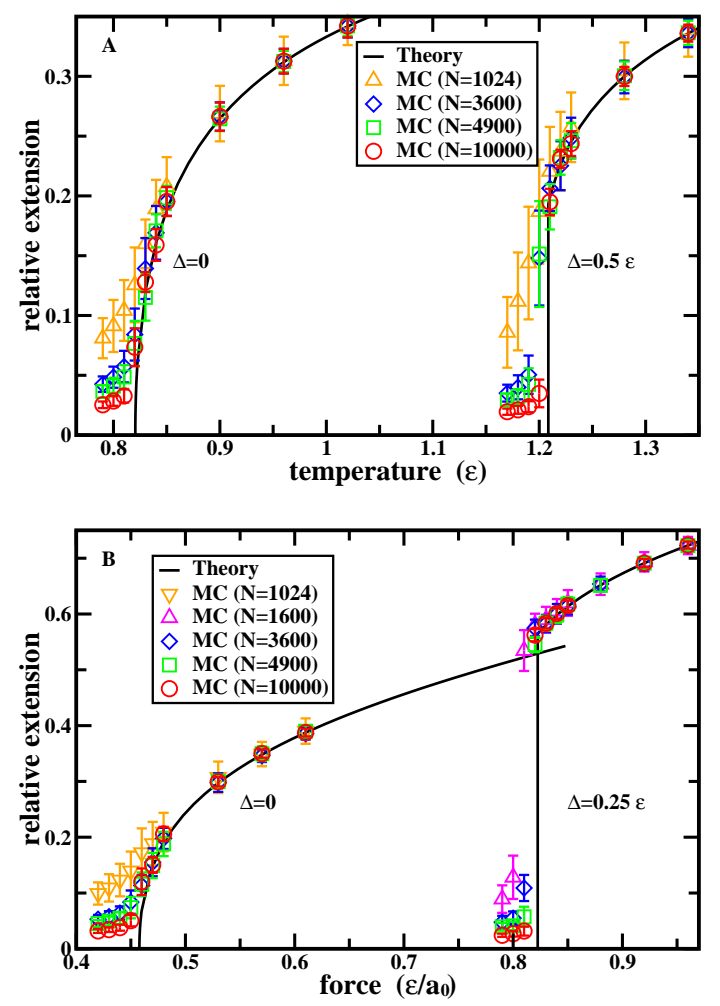

FIG. 2: (Color online) Response behavior of the polymer. (A) temperature-extension curve at force $f=0$; (B) Forceextension curve at temperature $T=0.590928 \epsilon$. Solid lines are analytical predictions and symbols are MC simulation results for different system sizes.
Results for $\Delta \equiv 0$. We perform analytical calculations as well as Monte Carlo simulations on the model system. The $\ell-T$ relation at $f=0$ is shown in Fig. 2 $\mathrm{a}$, and the $\ell-f$ relation at $T=0.591 \epsilon$ is shown in Fig. 2 $b$. When there is no external force, a second-order globulecoil phase transition occurs at $T_{\mathrm{gc}}(0)=0.8205 \epsilon$. The force-induced globule-coil transition at constant temperature is also second-order. Our findings are in accordance with Refs. [16, 18]. In the vicinity of the collapse transition, the relative extension of the extended coil phase can be expressed in a scaling form in terms of temperature change $\delta T$ or force change $\delta f$ :

$$
\begin{aligned}
\ell\left(T_{\mathrm{gc}}(f)+\delta T\right) & \approx \ell\left(T_{\mathrm{gc}}(f)\right)+c_{1}(\delta T)^{\gamma_{1}}, \\
\ell\left(f_{\mathrm{gc}}(T)+\delta f\right) & \approx \ell\left(f_{\mathrm{gc}}(T)\right)+c_{2}(\delta f)^{\gamma_{2}},
\end{aligned}
$$

where $T_{\mathrm{gc}}(f)$ and $f_{\mathrm{gc}}(T)$ are, respectively, the transition temperature at fixed force and the transition force at fixed temperature; $\gamma_{1}$ and $\gamma_{2}$ are two scaling exponents; and $c_{1}, c_{2}$ are two numerical constants. In the case of $\Delta \equiv 0$, we have $\ell\left(T_{\mathrm{gc}}(f)\right)=\ell\left(f_{\mathrm{gc}}(T)\right)=0$; and we find that the scaling exponent in Eq. (14) is independent of force with $\gamma_{1}=1 / 2$ (see Fig. 3), which is consistent with a second-order phase transition. The scaling relation Eq. (15) with $\gamma_{2}=1 / 2$ is also confirmed by numerical calculation (data not shown).

Results for $\Delta>0$. In this case, the collapse transition becomes first-order. For example at $\Delta=0.5 \epsilon$ and $f=0$, the relative extension jumps from zero to $\ell=0.193$ at the transition temperature $T_{\mathrm{gc}}(0)=1.209 \epsilon$ (Fig. 2 $2 \mathrm{a}$ ). Such a large jump is also observed in the force-induced transition (Fig. 2b). A non-zero bending stiffness therefore is able to dramatically enhance the cooperativity of the globule-coil phase transition. This may be partially understood in the following way. A positive bending energy significantly decreases the configurational entropy of a coil segment. Consequently the globule-coil transition will occur at higher temperature and higher force, and once the polymer is unfolded it favors those highly elongated configurations which have fewer bends.

As is expected for a first-order phase transition, in the case of $\Delta>0$, the scaling exponents in the scaling forms (14) and (15) are $\gamma_{1}=\gamma_{2}=1$ (Fig. 3).

Conclusion.-In this work we studied the collapse transition of a $2 \mathrm{D}$ partially directed lattice polymer model using the Lifson approach [24]. We devided each configuration of the polymer into $\beta$-sheet segments and coil segments and then calculated their partition functions separately. This approach enables us to precisely calculate the free energy density of the system. Previous tricritical field theory [22, 23] and numerical scaling analysis [18] predicted that the collapse transition of a flexible $(\Delta \equiv 0)$ partially directed lattice polymer is secondorder. We have confirmed this prediction. Our calculations further revealed that, the susceptibility $\chi=\partial \ell / \partial f$ of the polymer diverges as $\chi \propto\left(f-f_{\mathrm{gc}}(T)\right)^{-1 / 2}$ or 


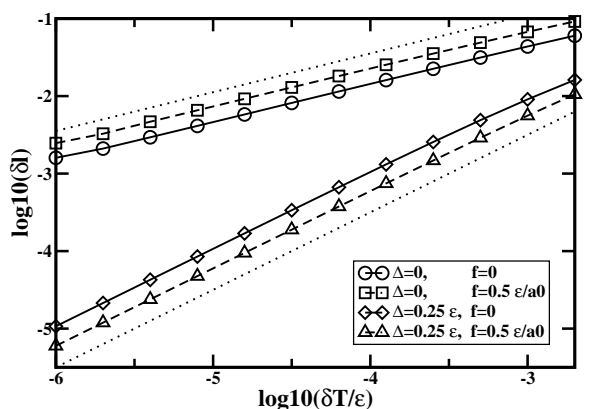

FIG. 3: The scaling relation between extension increment $\delta \ell$ $\left[\equiv \ell\left(T_{\mathrm{gc}}(f)+\delta T\right)-\ell\left(T_{\mathrm{gc}}(f)\right)\right]$ and $\delta T$. The upper and lower dotted line has slope $1 / 2$ and 1 , respectively. The dimension of the matrix $\Lambda(\zeta)$ is set to $L=5000$.

$\chi \propto\left(T-T_{\mathrm{gc}}(f)\right)^{-1 / 2}$ as $f$ or $T$ approaches the phase transition value from above. The most important result of the present paper is that, a non-zero bending stiffness of the polymer changes the nature of the collapse transition from being second-order to being first-order. This conclusion is in agreement with an earlier exact enumeration study 20]. It is well known that, in the 2D Ising model the paramagnet-ferromagnet phase transition changes from being second-order to being first-order under the action of a non-zero external magnetic field. It it is interesting that in the $2 \mathrm{D}$ polymer system such a qualitative change is caused not by an external field (such as the external force), but by an internal (microscopic) parameter, the bending stiffness $\Delta$.

At this point, it remains to be seen whether the above conclusion also holds if we remove the constraint of partial directedness. In the case of DNA thermal denaturation, including long-range excluded-volume interactions between different bubbles changes the order of the phase transition 26]. When additional long-range excludedvolume interactions are included, it may not be surprising if the temperature-induced 2D collapse transition will be first-order only when the bending energy is much larger than the contacting energy $\epsilon$ [21]. On the other hand, the force-induced 2D collapse transition may have different behavior. Mean-field work of Ref. [16] suggested that in the globule-coil transition, when a force is approached from below, a collapsed 2D polymer takes shape of an elongated elipse (i.e., partially directed). The mathematical constraint of partial directedness of the present paper may not qualitatively change the cooperativity of the force-induced 2D collapse transition. Additional MC simulation work as well as exact numerical enumeration study are needed to clarify this point. (When $\Delta=0.3 \epsilon$, our prelimilary $\mathrm{MC}$ results hinted at a first-order phase transition, with $f_{c} \approx 0.9 \epsilon$ at $T=0.6 \epsilon$.) It is also highly desirable to perform real $2 \mathrm{D}$ polymer collapse experiments. For example, a flexible or semiflexible biopolymer [such as DNA and poly(Glycine)] can be attached to a mobile lipid bilayer [19]. Polymer configurations can be recorded in real-time and manipulated by controlling temperature or external force.

We thank X.-S. Chen, D. Giri, P. Grassberger, D. Marenduzzo, H. Orland for critical comments, and the State Key Lab. of Sci. and Engn. Computing of CAS for computational facilities. HZ acknowledges the initial support of R. Lipowsky and the AvH foundation, SK acknowledges the hospitality of the MPIPKS-Dresden.

[1] J. des Cloizeaux and G. Jannink, Polymers in Solution (Clarendon Press, Oxford, UK, 1990).

[2] A. Y. Grosberg and A. R. Khokhlov, Statistical Physics of Macromolecules (American Institute of Physics, New York, 1994).

[3] C. Vanderzande, Lattice Models of Polymers (Cambridge Univ. Press, Cambridge, UK, 1998).

[4] P.-G. de Gennes, J. Physique Lett. 36, L55 (1975).

[5] M. J. Stephen, Phys. Lett. A 53, 363 (1975).

[6] B. Duplantier, J. Phys. (Paris) 43, 991 (1982).

[7] A. L. Owczarek and T. T. Prellberg, Europhys. Lett. 51, 602 (2000).

[8] C. Vanderzande, A. L. Stella, and F. Seno, Phys. Rev. Lett. 67, 2757 (1991).

[9] B. Duplantier, J. Stat. Phys. 110, 691 (2003).

[10] X. Wang, X. Qiu, and C. Wu, Macromolecules 31, 2972 (1998).

[11] K. Yoshikawa, M. Takahashi, V. V. Vasilevskaya, and A. R. Khokhlov, Phys. Rev. Lett. 76, 3029 (1996).

[12] F. Rampf, W. Paul, and K. Binder, Europhys. Lett. 70, $628(2005)$.

[13] H. Clausen-Schaumann, M. Seitz, R. Krautbauer, and H. E. Gaub, Curr. Opin. Chem. Biol. 4, 524 (2000).

[14] C. G. Baumann, V. A. Bloomfield, S. B. Smith, C. Bustamante, M. D. Wang, and S. M. Block, Biophys. J. 78, 1965 (2000).

[15] A. Halperin and E. B. Zhulina, Europhys. Lett. 15, 417 (1991).

[16] P. Grassberger and H.-P. Hsu, Phys. Rev. E 65, 031807 (2002).

[17] D. Marenduzzo, A. Maritan, A. Rosa, and F. Seno, Phys. Rev. Lett. 90, 088301 (2003).

[18] A. Rosa, D. Marenduzzo, A. Maritan, and F. Seno, Phys. Rev. E 67, 041802 (2003).

[19] B. Maier and J. O. Rädler, Phys. Rev. Lett. 82, 1911 (1999).

[20] S. Kumar and D. Giri, Phys. Rev. E 72, 052901 (2005).

[21] S. Doniach, T. Garel, and H. Orland, J. Chem. Phys. 105, 1601 (1996).

[22] R. Brak, A. J. Guttmann, and S. G. Whittington, J. Phys. A: Math. Gen. 25, 2437 (1992).

[23] A. L. Owczarek and T. Prellberg, Physica A 205, 203 (1994).

[24] S. Lifson, J. Chem. Phys. 40, 3705 (1964).

[25] See EPAPS Document No. E-PRLTAO-97-054602 for calculation details.

[26] Y. Kafri, D. Mukamel, and L. Peliti, Phys. Rev. Lett. 85, 4988 (2000). 